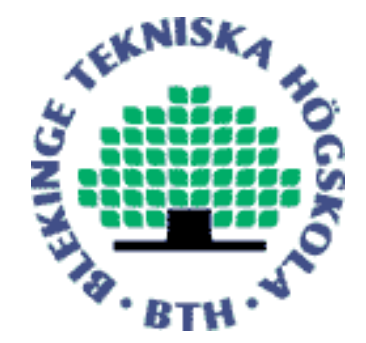

Copyright (C) 2012 IEEE.

Citation for the published paper:

Performance of Cognitive Radio Spectrum Access with Intra- and Inter-Handoff

Yong Yao, Said Rutabayiro Ngoga, David Erman, Adrian Popescu

International Conference on Communications ICC

2012 Ottawa

This material is posted here with permission of the IEEE. Such permission of the IEEE does not in any way imply IEEE endorsement of any of BTH's products or services Internal or personal use of this material is permitted. However, permission to reprint/republish this material for advertising or promotional purposes or for creating new collective works for resale or redistribution must be obtained from the IEEE by sending a blank email message to pubs-permissions@iee.org.

By choosing to view this document, you agree to all provisions of the copyright laws protecting it. 


\title{
Performance of Cognitive Radio Spectrum Access with Intra- and Inter-Handoff
}

\author{
Yong Yao, Said Rutabayiro Ngoga, David Erman, and Adrian Popescu \\ School of Computing, Blekinge Institute of Technology, 37179 Karlskrona, Sweden \\ Email: \{yya, srn, der, apo\}@bth.se
}

\begin{abstract}
Opportunistic spectrum access (OSA) is a technology that allows unlicensed users to access spectrum holes and to provide so efficient use of radio resources. Most studies done on OSA focus on the situation when the unlicensed user performs the spectrum handoff only within a single cognitive radio network (so-called intra-handoff). In this paper, we consider the users (licensed or unlicensed) to be able to do inter-handoff among different cognitive radio cells as well. The cells provide priority to inter-handoff users. By considering multiple cells being in steadystate and showing identical statistics, the arrival rates of interhandoff users are determined. We study the OSA performance of unlicensed users under both intra- and inter-handoff schemes, with respect to the blocking and forced-termination probabilities of unlicensed users as well as the unlicensed user servicecompletion and inter-handoff throughputs. Our Markov chain based numerical analysis is validated by simulation experiments.
\end{abstract}

Index Terms-Cognitive radio, opportunistic spectrum access, cellular networks, inter handoff, Markov chain.

\section{INTRODUCTION}

As a result of static frequency allocation policy, today's spectrum is rarely fully utilized in the domains time, frequency and geographical location [1]. Spectral under-utilization is contrasting the increasing demand for radio resources (i.e., spectrum channels) due to the rapid growth of wireless services and applications such as sensor networks and smartphones. This conflict reveals a serious problem of spectrum scarcity challenging inherently in $4 \mathrm{G}$ and beyond. To alleviate the problem, attractive technologies like Cognitive Radio (CR), first coined by Mitola in 1999 [2], have been advanced. In general, $\mathrm{CR}$ is envisioned to act as a highly intelligent radio where transmission parameters like, e.g., frequency range, transmit power and modulation type can be altered by learning radio environment.

In CR networks, the unlicensed users are equipped with CR devices. They are known as secondary users (SUs) and can use licensed spectrum as long as they do not harmfully interfere with licensed users (i.e., primary users or PUs). Given the dynamic nature of PU's activity, one feasible approach to implement CR networks is the Opportunistic Spectrum Access (OSA) model [3]. In this model, if and when a channel is not used by the PU, the channel is said to be available for the SU. Identification of the spatio-temporally available channels (also know as spectrum holes) can be done by spectrum sensing or by database based solutions [4]-[6]. Once acquiring available channels, SUs are allowed to opportunistically operate in them. To protect the PU transmission, the SUs must

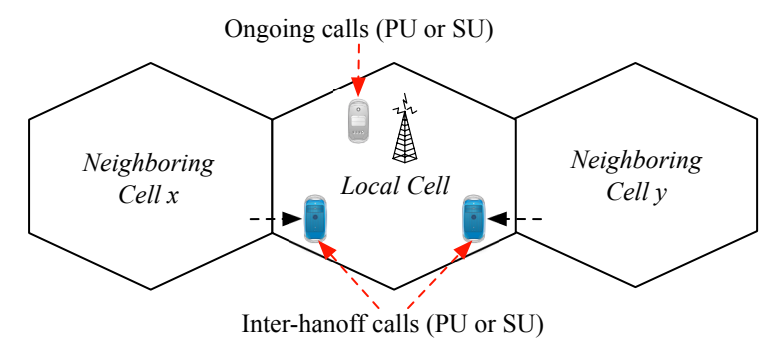

Fig. 1. CR networks with inter-handoff.

vacate accessed channels if the PUs start using them again. The PU protection mechanism, however, significantly affects the transmission performance of SUs. To study the related performance characteristics, the Markov chain based modeling approach is widely used in recent studies [7]-[11].

The authors of [7]-[11] use a model based on which every channel is divided into several sub-channels. All sub-channels in the channels not occupied by PUs are available for SUs. When a newly arrived PU occupies a channel, all SUs using this channel attempt to switch the transmission on other available sub-channels, which is known as spectrum handoff. Un-successful handoff creates the state of SU's transmission being terminated. In addition, if all channels are fully used by PUs and SUs, transmission requests from new SUs are blocked. In [7] [8], an infinite user population based loss queueing system is reported for these purposes. In [9], a finite buffer is introduced for queueing new SUs and it is used for decreasing blocking probability. In [10], a finite user population is adopted, so that the terminated SUs can return to the queue until they find other available sub-channels again. In [11], SUs are categorized into two classes with low-priory and high-priority, respectively. The goal is to accommodate more high-priority SUs when handoff occurs. In all studies mentioned above, the spectrum handoff is done by SUs, and in fact this indicates the intra-handoff meaning that switching transmission on different channels takes place inside a single CR network [4].

Here, cellular wireless communication networks are considered where inter-handoff calls may frequently occur in a local cell, as shown in Fig. 1. The inter-handoff calls mean the calls that are handed over from neighboring cells to the local cell. Furthermore, the local cell needs to serve these inter-handoff calls and offers priority to them [12] [13]. As a result, the 
Quality of Service (QoS) of ongoing calls (including previous inter-handoff calls) in the local cell would be impaired. To our best knowledge, there has been little investigation so far on the OSA performance in the presence of inter-handoff calls.

In this paper, a realistic CR network is considered in terms of a wireless cell that concurrently receives new calls initiated within the local cell and inter-handoff calls handed over by the neighboring cells. These calls are radio communications associated with either PUs or SUs. Two inter-handoff schemes are suggested to manage inter-handoff calls. The two interhandoff schemes deal with the PU and SU calls, respectively. Moreover, we consider a particular scenario that if multiple CR cells are in steady-state, these cells show identical statistics. Thus, the rate of inter-handoff calls arriving in a local cell should equal the rate of inter-handoff calls going out of the local cell for PU and SU calls, respectively [13]. This helps in determining the values of inter-handoff parameters. By employing Continuous Time Markov Chain (CTMC), we build a queuing model to represent OSA under the inter-handoff schemes. To evaluate the OSA performance, we use metrics in terms of probabilities of blocking and terminating SU calls, and SU service-completion and inter-handoff throughputs.

The rest of the paper is organized as follows. Section II describes the system model and inter-handoff schemes. The queueing analysis is presented in Section III. The algorithm of determining the PU and SU inter-handoff parameters is presented in Section IV. The numerical and simulation results are discussed in Section V. Finally, we conclude the paper in Section VI.

\section{System Model AND INTER-HANDOFF SCHEMES}

\section{A. System Model}

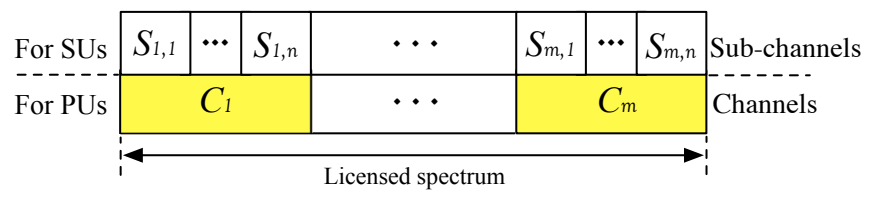

Fig. 2. System model.

An OSA based CR network is considered with $m$ identical channels, each consisting of $n$ identical sub-channels (see Fig. 2). Every channel is reserved for one $\mathrm{PU}$ at a time moment, while every sub-channel in the available channels can be accessed by one SU at a time moment. Similar to [7][11], we assume that there is a central controller responsible for allocating channels and sub-channels to PUs and SUs, respectively. We also assume the following.

New calls arrive independently in Poisson streams at mean rates $\lambda_{p 1}$ and $\lambda_{s 1}$ for PU and SU, respectively. The arrivals of inter-handoff calls independently follow Poisson processes with mean rates $\lambda_{p 2}$ and $\lambda_{s 2}$ for PU and SU, respectively. If either a new or an inter-handoff PU call is assigned to a channel, this call is said to become an ongoing PU call. If either a new or an inter-handoff SU call is assigned to a subchannel, this call is said to become an ongoing SU call.
The service times of ongoing calls completing service are exponentially distributed at mean rates $\mu_{p 1}$ and $\mu_{s 1}$ for PU and SU, respectively. In addition, the users (PU or SU) engaged in the call may depart the local cell. The departures independently follow exponential distributions with mean rates $\mu_{p 2}$ and $\mu_{s 2}$ for the PU and SU calls, respectively.

\section{B. Inter-handoff Schemes}

To give priority to inter-handoff calls, an inter-handoff scheme for PU calls and an intra/inter handoff scheme for SU calls are suggested. The two inter-handoff schemes are similar with the one used in [12] [13]. Differing from these works that only consider the licensed users inter-handoff, our suggested schemes mainly address the unlicensed users intra- and interhandoff under the licensed users protection mechanism.

1) Inter-handoff scheme for $P U$ calls: when an interhandoff PU call arrives and a channel is idle (not used by other PUs), the PU call is accepted and a channel is allocated to it. Otherwise, the inter-handoff PU call is blocked. When a new PU call arrives, it is accepted when at least $(g+1)$ channels are idle. Otherwise, the new PU call is blocked. In the case of $g>0$, the system gives priority to inter-handoff PU calls. Like in [13], $g$ is called the number of guard channels (for inter-handoff PU calls). To avoid new PU calls blocked altogether, $g$ must be less than $m$.

2) Intra/inter handoff scheme for SU calls: when an interhandoff SU call arrives and one or more available sub-channels are idle (not used by other SUs), the SU call is accepted and a sub-channel is allocated to it. Otherwise, the inter-handoff SU call is blocked. When a new SU call arrives, it is accepted if the number of idle sub-channels is not less than $(h+1)$. Otherwise, the new SU call is blocked. Similar to PUs, if $h>0$, the inter-handoff SU calls are prioritized. Here, $h$ is called the number of guard sub-channels for inter-handoff SU calls. For simplicity, we let $h$ be less than $n$. For intra-handoff, when an ongoing SU call is dropped due to PU channel occupancy and there exists an idle sub-channel, this sub-channel is allocated to the dropped SU call. Otherwise, the dropped SU call is terminated.

\section{QUeUing ANALYsis}

The queueing model is built up on the basis of CTMC, which has three characteristics: system state, state transition and steady-state probability. Let an integer pair $(i, j)$ denote a system state that $i$ ongoing SU calls and $j$ ongoing PU calls coexist within $n m$ sub-bands. Constrained by $(i+n j \leq n m)$, the state space is given by:

$$
S=\{(i, j) \mid i \in[0, n m], j \in[0, m],(i+n j) \in[0, n m]\}
$$

\section{A. State Transition}

The state transition of the system is triggered by three conditions: PU's activity, SU's activity without interruption by PUs, and SU's feedback in response to channel occupancy by PUs. The PU's activity includes i) accessing a channel, ii) releasing a channel at service-completion, and iii) the departure of ongoing PU calls. The SU's activity includes 
i) accessing a sub-channel, ii) SU releasing a sub-channel at service-completion, and iii) the departure of ongoing SU calls. The SU's feedback indicates the attempt of intra-handoff.

1) PU's activity: when there are $j$ ongoing PU calls in the system, let $\lambda_{j}^{(p)}$ and $\mu_{j}^{(p)}$ denote the PU call incoming rate (accessing a channel) and leaving rate (releasing a channel or departing the local cell), respectively. With regard to PUs inter-handoff scheme, $\lambda_{j}^{(p)}$ and $\mu_{j}^{(p)}$ are given by:

$$
\begin{aligned}
\lambda_{j}^{(p)} & = \begin{cases}\lambda_{p 1}+\lambda_{p 2}, & 0 \leq j \leq m-g-1 \\
\lambda_{p 2}, & m-g \leq j<m \\
0, & \text { others }\end{cases} \\
\mu_{j}^{(p)} & = \begin{cases}j\left(\mu_{p 1}+\mu_{p 2}\right), & 0<j \leq m \\
0, & \text { others }\end{cases}
\end{aligned}
$$

2) SU's activity without interruption by PUs: when no PU call is arriving and there are $i$ ongoing SU calls and $j$ ongoing PU calls in the system, an incoming SU call (new or interhandoff) is treated in three ways:

- If $(i+n j)<(n m-h)$, a sub-channel is allocated to an either new or inter-handoff SU call.

- If $(n m-h) \leq(i+n j)<m n$, a sub-channel is only allocated to an inter-handoff SU call.

- if $(i+n j)=n m$, no sub-channel is allocated to any new or inter-handoff SU calls.

Let $\lambda_{i, j}^{(s)}$ and $\mu_{i, j}^{(s)}$ denote the incoming (accessing a subchannel) and leaving (releasing a sub-channel or departing the local cell) rates of SU calls, respectively. Based on SUs intraand inter-handoff scheme, $\lambda_{i, j}^{(s)}$ and $\mu_{i, j}^{(s)}$ are given by:

$$
\begin{aligned}
& \lambda_{i, j}^{(s)}= \begin{cases}\lambda_{s 1}+\lambda_{s 2}, & i+n j \leq n m-h-1 \\
\lambda_{s 2}, & n m-h \leq i+n j<n m \\
0, & \text { others }\end{cases} \\
& \mu_{i, j}^{(s)}= \begin{cases}i\left(\mu_{s 1}+\mu_{s 2}\right), & (i, j) \in S, i \neq 0 \\
0, & \text { others }\end{cases}
\end{aligned}
$$

3) SU's feedback in response to channel occupancy: consider that at state $(i, j)$, an incoming PU call preempts a channel. The SUs using this particular channel are dropped and they attempt the intra-handoff. Depending on the total number of ongoing calls, the dropped SU calls experience either successful intra-handoff or forced-termination.

If $(i+n j) \leq(n m-n)$, every dropped SU call can find a new idle sub-channel. In other words, no ongoing SU calls are terminated. This results in the system state changed as $(i, j+1)$. However, if $(i+n j)>(n m-n)$, there are not enough idle sub-channels to be allocated to all dropped SU calls. Thus, some dropped SU calls are forced to be terminated. Let $s$ indicate the number of terminated SU calls, i.e., $r=$ $(i+n j)-(n m-n)$ and $r \in[1, n]$. Thus, the number of remaining ongoing SU calls is given by $(i-r)=n(m-j-$ $1)$. This indicates that the system changes state from $(i, j)$ to $(n(m-j-1), j+1)$. The corresponding transition rate is equal to $\lambda_{j}^{(p)}$, relying on the number of ongoing PU calls.

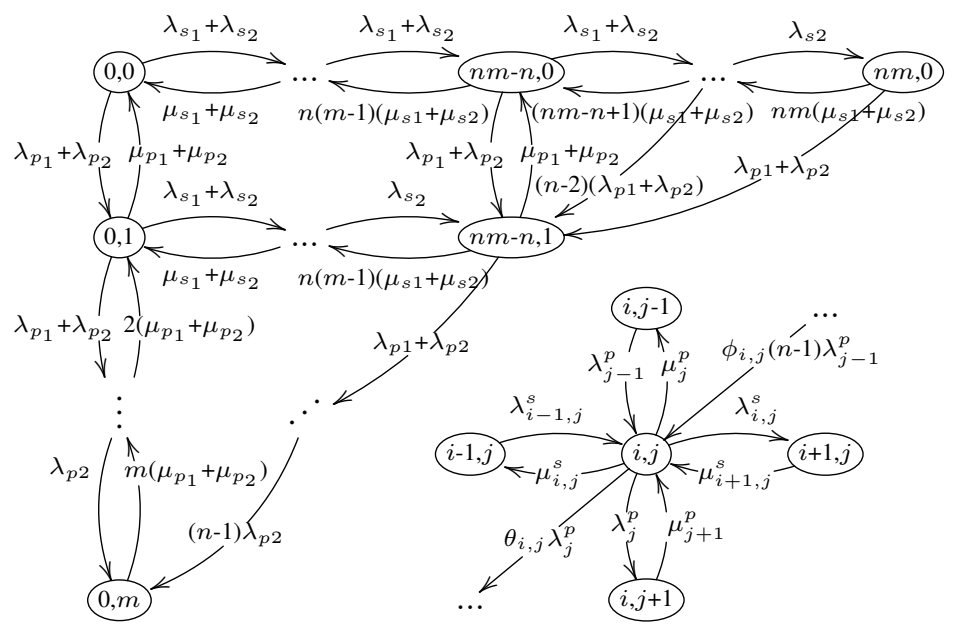

Fig. 3. State diagram for the modeled system, $g>0, h>0$.

\section{B. Steady-State Probability}

The state diagram for the modeled system is shown in Fig 3. Let $\pi_{i, j}$ denote the steady-state probability of state $(i, j)$. From the diagram, we write the steady-state balance equations:

$$
\begin{aligned}
& \pi_{i, j}\left(\lambda_{i, j}^{(s)}+\mu_{i, j}^{(s)}+\lambda_{j}^{(p)}+\mu_{j}^{(p)}+\theta_{i, j} \lambda_{j}^{(p)}\right) \\
= & \pi_{i-1, j} \lambda_{i-1, j}^{(s)}+\pi_{i+1, j} \mu_{i+1, j}^{(s)}+\pi_{i, j-1} \lambda_{j-1}^{(p)} \\
+ & \pi_{i, j+1} \mu_{j+1}^{(p)}+\phi_{i, j} \sum_{k=1}^{n}\left(\pi_{i+k, j-1} \lambda_{j-1}^{(p)}\right)
\end{aligned}
$$

where $\pi_{i, j}$ is equal to zero if $(i, j) \notin S, \theta_{i, j}$ is equal to one if $(j \neq m,(i+n j) \in(n m-n, n m])$ and zero if others, and $\phi_{i, j}$ is equal to one if $(i+n j=n m)$ and zero if others. We sum up all steady-state probabilities in conjunction with normalization constraint

$$
\sum_{\forall i, j}\left[\pi_{i, j} \mid(i, j) \in S\right]=1
$$

By combining the equations (4) and (5), we can construct a set of linear equations. By solving these linear equations, we can compute the steady-state probabilities of all states.

\section{Performance Metrics}

To explore the performance of SU calls under the suggested handoff schemes, we adopt five performance metrics. These are blocking probabilities of new and inter-handoff SU calls, forced-termination probability of ongoing SU calls, SU service-completion and inter-handoff throughputs.

1) Blocking probability: the incoming SU calls consist of two types, namely, new SU call and inter-handoff SU call. According to subsection III-A2, the case of blocking new SU calls occurs for $(i+n j) \geq(n m-h)$, and the case of blocking inter-handoff SU calls occurs for $(i+n j)=n m$. We denote the blocking probabilities of new and inter-handoff SU calls by $P_{b l}^{(1)}$ and $P_{b l}^{(2)}$, respectively. We then obtain:

$$
\begin{aligned}
& P_{b l}^{(1)}=\sum_{\forall i, j}\left[\pi_{i, j} \mid(i, j) \in S, i+n j \geq n m-h\right] \\
& P_{b l}^{(2)}=\sum_{\forall i, j}\left[\pi_{i, j} \mid(i, j) \in S, i+n j=n m\right]
\end{aligned}
$$




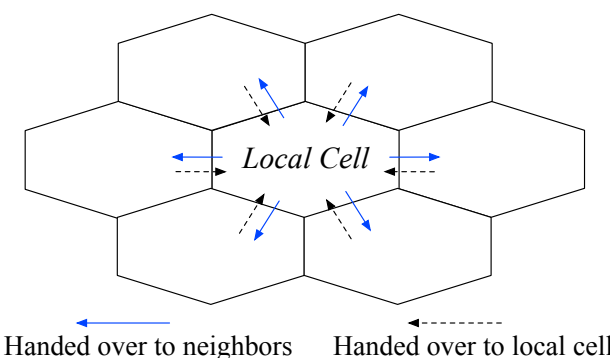

Fig. 4. Inter-handoff calls (PU or SU) handed over by either a local cell or its neighboring cells.

2) Forced-termination probability: as described in subsection III-A3, an arrived PU call forces the ongoing SU calls in the same channel to be dropped. The case of terminating a dropped SU call happens when the system can not accommodate the dropped SU call because of un-successful intra-handoff, i.e., $(i+n j)>(n m-n)$. To compute the forced-termination probability, denoted by $P_{f t}$, we divide the termination rate by the effective rate of accepting SU calls [8]. Given that $r=[(i+n j)-(n m-n)]$ ongoing SU calls are terminated because of the incoming PU call with rate $\lambda_{j}^{(p)}$, the termination rate is equal to $\left(r \lambda_{j}^{(p)} \pi_{i, j}\right)$. Furthermore, the acceptance rate is given by $\left(\lambda_{s 1}\left(1-P_{b l}^{(1)}\right)+\lambda_{s 2}\left(1-P_{b l}^{(2)}\right)\right)$. Therefore, the forced-termination probability of ongoing SU calls is formulated as:

$$
P_{f t}=\sum_{\forall i, j}^{\substack{(i, j) \in S \\ i+n j>m n-n}}\left[\frac{[(i+n j)-(n m-n)] \lambda_{j}^{(p)} \pi_{i, j}}{\lambda_{s 1}\left(1-P_{b l}^{(1)}\right)+\lambda_{s 2}\left(1-P_{b l}^{(2)}\right)}\right]
$$

3) Service-completion and inter-handoff throughput: the SU service-completion throughput traditionally indicates the average rate of SU calls completing the service. The SU interhandoff throughput indicates the average rate of ongoing SU calls out from the local cell (handed over by local cell to neighboring cells). Let $R_{\text {se }}$ and $R_{\text {inter }}$ denote the SU servicecompletion and inter-handoff throughputs, respectively. The expressions are given by $R_{s e}=\mu_{s 1} \sum_{\forall i, j}^{(i, j) \in S}\left[i \pi_{i, j}\right]$ and $R_{\text {inter }}=\mu_{s 2} \sum_{\forall i, j}^{(i, j) \in S}\left[i \pi_{i, j}\right]$.

\section{Determination of Inter-HandofF PARAmeters}

So far, we have assumed that all aforementioned parameters are priorly known. However, in reality, the PU inter-handoff arrival rate $\lambda_{p 2}$ depends on four parameters. They are the new PU arrival rate $\lambda_{p 1}$, PU service-completion rate $\mu_{p 1}$, departure rate of ongoing PU calls $\mu_{p 2}$ and PU inter-handoff priority $g$ [13]. For SUs, the inter-handoff arrival rate $\lambda_{s 2}$ depends not only on PU parameters $\lambda_{p 1}, \mu_{p 1}, \mu_{p 2}$ and $g$, but also on SU parameters $\lambda_{s 1}, \mu_{s 1}, \mu_{s 2}$ and $h$.

To determine $\lambda_{p 2}$ and $\lambda_{s 2}$, we suppose that all CR cells (local cell and its neighboring cells) show identical statistics. In other words, for the local CR cell being in steady-state, the inter-handoff arrival rate and the inter-handoff throughput should equal each other for PU and SU calls, respectively (Fig. 4). Since the PUs can exclusively occupy the channels (even

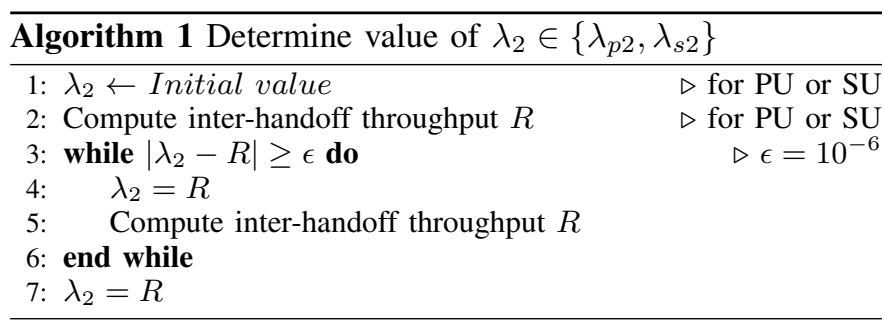

used by SUs), we apply the birth-death model for modeling the PU calls. Let $p_{r}(k)$ denote the steady-state probability of the $k$ ongoing PU calls existent in the system. According to the representation in [13], we obtain the balance functions as:

$$
\begin{aligned}
& 1 / p_{r}(0)=\sum_{k=0}^{m-g-1} \alpha(k, m, g)+\sum_{k=m-g}^{m} \beta(k, m, g) \\
& p_{r}(k)=\left\{\begin{array}{cc}
\alpha(k, m, g) p_{r}(0), & 0<k \leq m-g \\
\beta(k, m, g) p_{r}(0), & m-g \leq k \leq m
\end{array}\right.
\end{aligned}
$$

where,

$$
\begin{aligned}
\alpha(k, m, g) & =\frac{1}{k !} \cdot \frac{\left(\lambda_{p 1}+\lambda_{p 2}\right)^{k}}{\left(\mu_{p 1}+\mu_{p 2}\right)^{k}} \\
\beta(k, m, g) & =\frac{1}{k !} \cdot \frac{\left(\lambda_{p 1}+\lambda_{p 2}\right)^{m-g} \lambda_{p 2}^{k-(m-g)}}{\left(\mu_{p 1}+\mu_{p 2}\right)^{k}}
\end{aligned}
$$

Then, the PU inter-handoff throughput, denoted by $R_{i n t e r}^{p}$, is equal to $\mu_{p 2} \sum_{k=1}^{m}\left[k p_{r}(k)\right]$. Further, the inter-handoff PU calls are constrained by fixed-point equation $\lambda_{p 2}=R_{\text {inter }}^{p}$. Similarly, for inter-handoff SU calls, we have the constraint $\lambda_{s 2}=R_{\text {inter }}$. Finally, determining the values of $\lambda_{p 2}$ and $\lambda_{s 2}$ follows an iteration method described by Algorithm 1 .

\section{Performance Evaluation}

In this section, we report numeric and simulation results for performance evaluation of OSA in the presence of inter-handoff. According to [7]-[9] and [11], we consider the following parameters: $m=3, n=6, \lambda_{p 1} \in$ $\{0.03,0.06,0.09,0.12\}, \mu_{p 1}=0.06, \mu_{p 2}=0.04, \lambda_{s 1}=0.82$, $\mu_{s 1}=0.68, \mu_{s 2}=0.45$, and $g \in\{1,2\}, h \in\{1,2\}$.

The simulation experiment is conducted to demonstrate the validity of the theoretical analysis. The simulator is developed in $\mathrm{C}++$. In the experiment, the simulator is run in a looping manner and each loop indicates $1 \mathrm{~ms}$. For every specific parameter setting, the simulator runs $t=10^{7} \mathrm{~s}$ simulation time. Considering a simulation run, we let $x, x^{\prime}, y, y^{\prime}, z, z^{\prime}$ and $w$ denote the numbers of arrived new/inter-handoff SU calls, blocked new/inter-handoff SU calls, terminated/completed SU calls and departures of ongoing SU calls, respectively. $P_{b l}^{(1)}$, $P_{b l}^{(2)}, P_{f t}, R_{s e}$ and $R_{\text {inter }}$ are expressed by $y / x, y^{\prime} / x^{\prime}$, $z /\left(x+x^{\prime}-y-y^{\prime}\right), z^{\prime} / t$ and $w / t$, respectively.

Table I shows the computed values of inter-handoff parameters $\lambda_{p 2}$ and $\lambda_{s 2}$, and the SU inter-handoff throughput $R_{\text {inter }}$ obtained from simulations. The results of blocking probability of new SU calls $P_{b l}^{(1)}$, blocking probability of inter-handoff calls $P_{b l}^{(2)}$, forced-termination probability of ongoing SU $P_{f t}$ 
TABLE I

THE COMPUTED INTER-HANDOFF ARRIVAL RATES $\lambda_{p 2}$ AND $\lambda_{s 2}$; VALUES (OBTAINED FROM SIMULATIONS) OF SU INTER-HANDOFF THROUGHPUT $R_{i n t e r}$ ARE IN PARENTHESES.

\begin{tabular}{|l|c|c|c|c|c|c|}
\hline \multirow{2}{*}{$\lambda_{p 1}$} & \multicolumn{2}{|c|}{$\lambda_{p 2} \times 10^{2}$} & \multicolumn{4}{|c|}{$\lambda_{s 2} \times 10^{2}\left(R_{\text {inter }} \times 10^{2}\right)$} \\
\cline { 2 - 7 } & $g=1$ & $g=2$ & $g=1, h=1$ & $g=1, h=2$ & $g=2, h=1$ & $g=2, h=2$ \\
\hline 0.03 & 1.9564 & 1.9377 & $53.6784(53.6708)$ & $53.5908(53.5854)$ & $54.0257(54.0373)$ & $53.9894(53.9889)$ \\
\hline 0.06 & 3.5822 & 3.3746 & $51.7316(51.7329)$ & $51.5348(51.5211)$ & $53.2888(53.3260)$ & $53.2052(53.2374)$ \\
\hline 0.09 & 4.7516 & 4.2433 & $49.4642(49,4864)$ & $49.2005(49.2060)$ & $52.5459(52.5487)$ & $52.4318(52.4746)$ \\
\hline 0.12 & 5.5786 & 4.7920 & $47.4424(47.4629)$ & $47.1409(47.1097)$ & $51.9469(51.9897)$ & $51.8133(51.8413)$ \\
\hline
\end{tabular}

TABLE II

NumericAl AND SIMULATION (IN PARENTHESES) RESULTS OF BLOCKING PROBABILITy OF INTER-HANDOFF SU CALLS, $P_{b l}^{(2)}$.

\begin{tabular}{|c|c|c|c|c|}
\hline \multirow{2}{*}{$\lambda_{p 1}$} & \multicolumn{4}{|c|}{$P_{b l}^{(2)} \times 10^{2}$ (simulation $)$} \\
\cline { 2 - 5 } & $g=1, h=1$ & $g=1, h=2$ & $g=2, h=1$ & $g=2, h=2$ \\
\hline 0.03 & $0.4963(0.4893)$ & $0.4943(0.4849)$ & $0.2016(0.2005)$ & $0.2008(0.2001)$ \\
\hline 0.06 & $2.2259(2.2283)$ & $2.2214(2.2287)$ & $0.8456(0.8539)$ & $0.8436(0.8486)$ \\
\hline 0.09 & $4.3263(4.3065)$ & $4.3204(4.3014)$ & $1.5075(1.5121)$ & $1.5049(1.4840)$ \\
\hline 0.12 & $6.2711(6.2658)$ & $6.2644(6.2834)$ & $2.0488(2.0284)$ & $2.0457(2.0550)$ \\
\hline
\end{tabular}

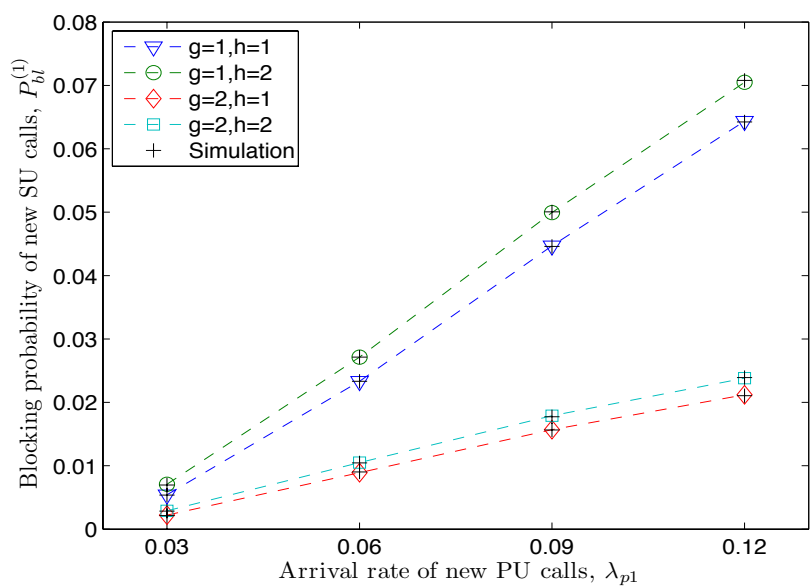

Fig. 5. Blocking probability of new SU calls $P_{b l}^{(1)}$ versus $\lambda_{p 1}$.

and SU service-completion throughput $R_{s e}$ are shown in Figs. $5,6,7$, and 8 , respectively. In each of the figures, the marker ' + ' indicates the simulation results. In Figs. 6 and 7 , we observe that, for fixed PU priority $g(=1$ or $=2)$, the values of $P_{b l}^{(2)}$ and $P_{f t}$ under $h=1$ differ only slightly from the ones under $h=2$, respectively. Therefore, we additionally report the results of $P_{b l}^{(2)}$ and $P_{f t}$ in Tables II and III, respectively. From the tables and figures, we observe that the simulation results closely match the analytical results. The discussion about OSA performance is as follows.

For fixed $g$, Table I shows that the PU inter-handoff arrival rate $\lambda_{p 2}$ increases with the new PU arrival rate $\lambda_{p 1}$. The reason for this is intuitive. The larger the number of new PU calls in a cell is, the larger the departure number of ongoing PU calls (related to PU inter-handoff throughput) becomes.

For fixed $g$ and fixed SU priority $h(=1$ or $=2)$, Table I shows that the SU inter-handoff arrival rate $\lambda_{s 2}$ decreases with $\lambda_{p 1}$. This is because the SUs' competition for channel utilization with PUs is enhanced when $\lambda_{p 1}$ is increasing. The

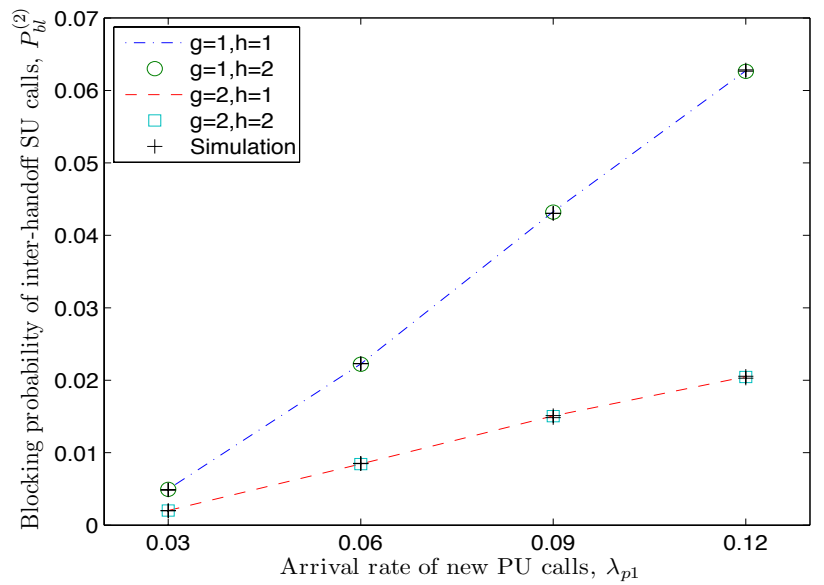

Fig. 6. Blocking probability of inter-handoff SU calls $P_{b l}^{(2)}$ versus $\lambda_{p 1}$.

enhancement can be observed from Figs. 5, 6, 7. That is, if both $g$ and $h$ are fixed, $P_{b l}^{(1)}, P_{b l}^{(2)}$ and $P_{f t}$ increase with $\lambda_{p 1}$. As such, $R_{s e}$ also decreases with $\lambda_{p 1}$, as shown in Fig. 8.

If both $\lambda_{p 1}$ and $g$ are fixed, Fig. 5 shows that $P_{b l}^{(1)}$ increases with $h$ (from $h=1$ to $h=2$ ). On the contrary, Table II and III show that both $P_{b l}^{(2)}$ and $P_{f t}$ decrease with $h$. Furthermore, by comparing Fig. 5 with Figs. 6 and 7, we observe that the amount of increase in $P_{b l}^{(1)}$ is larger than the ones of decrease in $P_{b l}^{(2)}$ and in $P_{f t}$. Hence, $R_{s e}$ decreases with $h$ (see Fig. 8).

Moreover, for fixed $\lambda_{p 1}$, it is clear that the number of blocked new PU calls under $g=2$ is larger than the one under $g=1$. This reduces the PU inter-handoff throughput so as to decrease $\lambda_{p 2}$, as shown in Table I.

For SUs, if both $\lambda_{p 1}$ and $h$ are fixed, the SUs under $g=2$ can acquire much more access opportunities to use available channels, as compared with the situation under $g=1$. Therefore, $P_{b l}^{(1)}, P_{b l}^{(2)}$ and $P_{f t}$ decrease with $g$, and thus $R_{s e}$ is improved, as shown in Figs. 5, 6, 7, and 8, 
TABLE III

NUMERICAL AND SIMULATION (IN PARENTHESES) RESUlTS OF FORCED-TERMINATION PROBABILITY OF ONGOING SU CALLS, $P_{f t}$.

\begin{tabular}{|c|c|c|c|c|}
\hline \multirow{2}{*}{$\lambda_{p 1}$} & \multicolumn{4}{|c|}{$P_{f t} \times 10^{2}($ simulation $)$} \\
\cline { 2 - 5 } & $g=1, h=1$ & $g=1, h=2$ & $g=2, h=1$ & $g=2, h=2$ \\
\hline 0.03 & $0.1294(0.1289)$ & $0.1280(0.1252)$ & $0.0524(0.0511)$ & $0.0518(0.0505)$ \\
\hline 0.06 & $0.5853(0.5861)$ & $0.5803(0.5824)$ & $0.2194(0.2210)$ & $0.2172(0.2166)$ \\
\hline 0.09 & $1.1539(1.1500)$ & $1.1459(1.1408)$ & $0.3917(0.3896)$ & $0.3880(0.3848)$ \\
\hline 0.12 & $1.6981(1.6922)$ & $1.6881(1.6883)$ & $0.5333(0.5297)$ & $0.5285(0.5286)$ \\
\hline
\end{tabular}

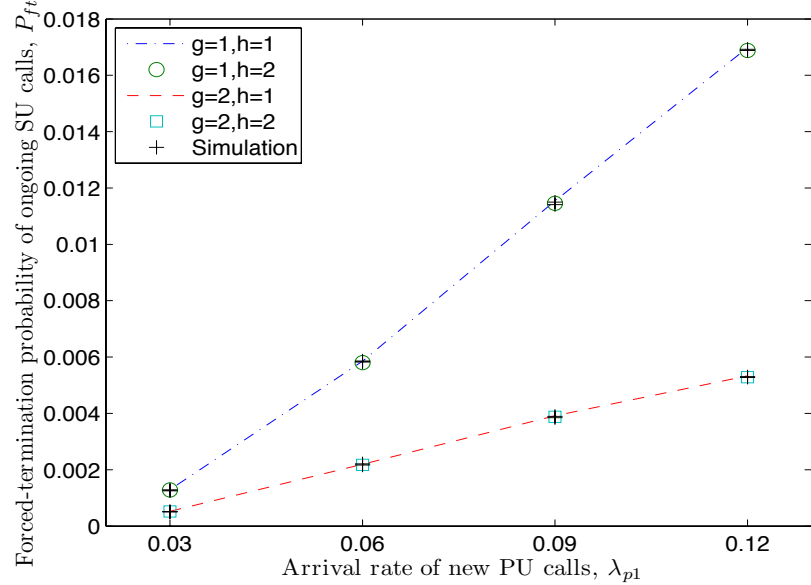

Fig. 7. Forced-termination probability of ongoing SU calls $P_{f t}$ versus $\lambda_{p 1}$.

respectively. For instance, Fig. 8 shows that the value of $R_{s e}$ under $g=2, h=1, \lambda_{p 1}=0.12$ is about $9 \%$ larger than the one under $g=1, h=1, \lambda_{p 1}=0.12$.

\section{CONCLUSION}

The opportunistic spectrum access in cognitive radio networks with user inter-handoff among different wireless cells has been studied. Two inter-handoff schemes have been suggested to give priorities to inter-handoff PU and SU calls, respectively. A continuous time Markov chain based queueing model was used to investigate the transmission performance of SUs with intra- and inter-handoff activities. The steadystate of multiple cells showing identical statistics has been also considered, based on which the inter-handoff arrival rates of PUs and SUs are determined. For performance evaluation, we derived blocking probabilities of new and inter-handoff SU calls, forced-termination probability of ongoing SU calls, SU service-completion throughput, and SU inter-handoff throughput. An interesting result is that the SU service-completion throughput can be increased by increasing priority level of inter-handoff PUs. The analytical results were validated by simulation results.

\section{REFERENCES}

[1] FCC Spectrum Policy Task Force, "Report of the spectrum efficiency working group", Technical Report 02-135, Federal Communications Commission, Washington, D.C., 2002.

[2] J. Mitola III, "Cognitive radio: an integrated agent architecture for software defined radio", Ph.D. Dissertation, KTH, Stockholm, 2000.

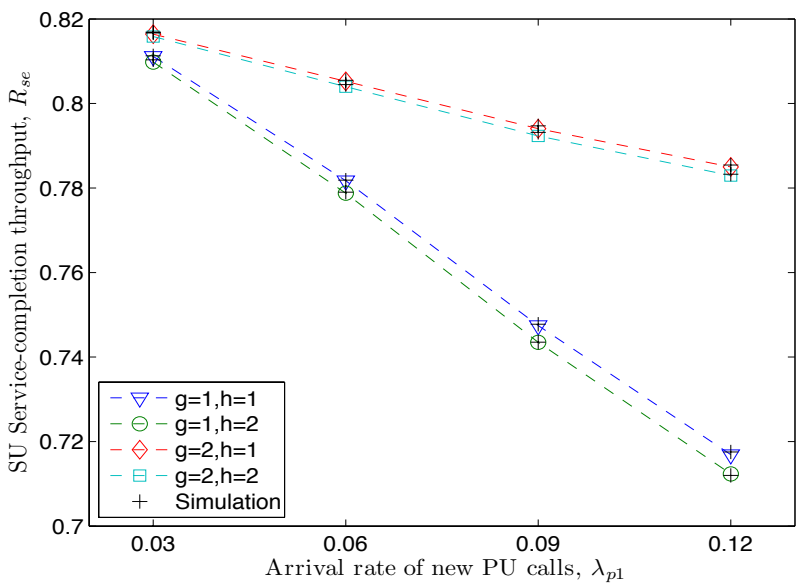

Fig. 8. SU service-completion throughput $R_{s e}$ versus $\lambda_{p 1}$.

[3] Q. Zhao, L. Tong, A. Swami, and Y. Chen "Decentralized cognitive MAC for opportunistic spectrum access in ad hoc networks: a POMDP framework", IEEE Journal on Selected Areas in Communications, vol. 25, no. 3, pp. 589-600, Apr. 2007.

[4] I.F. Akyildiz, W.-Y. Lee, M.C. Vuran, and S. Mohanty, "A survey on spectrum management in cognitive Radio Networks", IEEE Соттиnications Magazine, vol. 46, issue 4, pp. 40-48, Apr. 2008.

[5] W.-Y. Lee, K.R. Chowdhury, and M.C. Vuran, "Spectrum sensing algorithms for cognitive radio networks", Cognitive Radio Networks, pp. 3-35, Auerbach Publications, 2009.

[6] A.O. Popescu, D. Erman, M. Fiedler, A.P. Popescu, and D. Kouvatsos, "A middleware framework for communication in cognitive radio networks", International Congress on Ultra Modern Telecommunications and Control Systems, Moscow, Russia, Oct. 2010.

[7] X. Zhu, L. Shen, and T.-S. P. Yum, "Analysis of cognitive radio spectrum access with optimal band reservation", IEEE Communications Letters, vol. 11, no. 4, pp. 304-306, Apr. 2007.

[8] W. Ahmd, J. Gao, H.A. Suraweera, and M. Faulkner, "Comments on 'Analysis of cognitive radio spectrum access with optimal band reservation' ", IEEE Transition on Wireless Communication, vol. 8, no. 9 , pp. 4488-4491, Sep. 2009.

[9] Y. Zhang, "Dynamic spectrum access in cognitive radio wireless network" IEEE International Conference on Communications, Beijing, China, May. 2008.

[10] E.W.M. Wong and C.H. Foh, "Analysis of cognitive radio spectrum access with finite user population", IEEE Communications Letters, vol. 13, no. 5, pp. 294-296, May. 2009.

[11] V.K. Tumuluru, P. Wang, and D. Niyato, "Performance analysis of cognitive radio spectrum access with prioritized traffic", IEEE International Conference on Communications, Kyoto, Japan, Jun. 2011.

[12] D. Niyato, E. Hossain, and A.S. Alfa, "Performance analysis of multiservice wireless cellular networks with MMPP call arrival patterns", IEEE Global Communications Conference, Dallas, USA, Nov. 2004.

[13] K.S. Trivedi, Probability and statistics with reliability, queuing, and computer science applications, John Wiley \& Sons, Inc., 2002. 